\title{
Mathematik und Weltläufigkeit
}

\author{
carmonent
}

\section{Mathematik als Sprache}

Mathematik ist eine Sprache, in der sich Vollkommenes und Schönes ausdrücken lässt. Diese Sprache muss man lernen. Das wird nicht leicht gemacht. Diejenigen, die sie verstehen, bleiben vorwiegend notgedrungen unter sich.

Mathematik ist eine Sprache, mit der sich vieles Nützliche ausdrücken lässt. Dazu muss die Mathematik sich in ein Sprachgemisch anderer Sprachen einfügen (wie diese anderen auch) und kann dann eine wichtige Rolle spielen. Sie weigert sich aber unbewusst oder bewusst, „nur" als Fremdworttupfer in anderen Sprachen herzuhalten. Sie bemüht sich nicht, sich flüssig einzufügen.

Das Erlernen der mathematischen Sprache ist eine Leistung an sich. Das Durchdringen komplexer Theoriegebäude ist eine Leistung an sich, deren Bewältigung Glücksgefühle erzeugt und das nun nicht mehr nur verschwommen wahrgenommene Gebäude oft schöner erscheinen lässt, als es sein mag. Da das Verstehen so schwer ist und deshalb so im Vordergrund steht, ist das Sprechen wichtiger als das Bemühen, etwas Wichtiges zu sagen.

Deshalb sagt Mathematik vielen auch dann nichts, wenn Sie es denn verstünden. Mathematik ist anderen wie das Chinesische so fern und muss wohl in böhmischen Dörfern gesprochen werden. So sehen es viele, was die Mathematiker trotzig und halbherzig betrauern.

\section{Wie soll man Sprachen erlernen?}

Wir lernen eine Sprache doch eigentlich, um unser Denken um einen Kulturkreis zu erweitern oder etwa, um einen wirtschaftlichen Nutzen daraus zu ziehen. Wir tun das also, weil wir die noch ferne Kultur lieben oder weil der wirtschaftliche Nutzen für uns ganz greifbar ist. Warum lernen so wenige Latein? Weil die Liebe zum Altertum nicht mehr so verbreitet ist und weil sich das Altertum heute anders als mit der lateinischen Sprache erschließen lässt.
Warum lernen fast alle so ungern die Sprache der Mathematik? Weil ihre Vertreter die Liebe zu ihr erst nach dem Erlernen der Sprache verheißen - und die Liebe nicht vor das Erlernen zu setzen vermögen. Das kann die Astronomie. Mein Herz kann die Sterne erfassen ohne alle die Formeln. Das kann die Physik, die Chemie, die Biologie. Das können so viele der Wissenschaften. Manche auch nicht! Erinnern Sie sich, als vor einigen Jahrzehnten die Soziologen und Psychologen mit unverständlichem Kauderwelsch so sehr Sprachnebel um sich warfen, dass wir nur finstere Profilierungssucht darin sahen? Diese Wissenschaften hatten damals vergessen, dass sie die Aufgabe haben, uns etwas Wichtiges zur Seele und zur Gemeinschaft zu sagen. Sehen Sie nicht auch, wie die Wirtschaftswissenschaften nur dem Shareholder zu dienen scheinen und damit Unterdrückungswerkzeuge ersinnen, die den Wohlstand der Nationen aus dem Auge verloren haben? Sehen Sie, dass die Philosophie so speziell und komplex geworden ist, dass sie nicht mehr zum Leben des Normalmenschen beiträgt?

Eine lebendige Sprache erfreut einen ganzen Kulturkreis, der diese Sprache liebt, weil sie ihm Wichtiges für Herz, Geist und materielles Leben zu geben vermag. Dann erlernt man sie gern. Wer sie aber gerne erlernen möchte, die neue Sprache etwa der Mathematik, sollte mit offenen Armen empfangen werden. Das eben tut die Mathematik nicht. Der Student gerät in eine Universität, die konsequent von Anfang an die normale Sprache spricht - mehr nicht. Keine wirkliche Erklärung der Zeichen. Keine Einführung in eine Grammatik. Keine Einführung in die wichtigsten Vokabeln. Keine Babysprache, die wir bei Kindern benutzen. Rücksichtslos sprechen alle Eingeweihten Mathematik zu Verzweifelten. Diejenigen, die Mathematik sprechen, waren einst auch verzweifelt, bissen sich aber durch. Sie wissen, dass sich die Nebel nach einigen Jahren legen, wahrscheinlich nicht vor dem Bachelor. „Da muss man durch!" (Ich erinnere mich, wie der Unteroffizier auf den Vorwurf des Quälens antwortete: „Das muss sein. Auch ich wurde gequält.") 
Neulich war eine Amerikanerin in Frankreich im TV zu sehen. „Ich kann nur ein paar Wörter hier sprechen, ich komme mir so dumm vor. Das macht mich schon scheu. Aber die Franzosen, die es natürlich merken, geben mir dann ganz brutal das Gefühl, wirklich dumm zu sein. Ich leide hier." Da dachte ich an die Telefonkonferenzen bei IBM, wo die Amerikaner inzwischen global in der Minderheit sind, aber so schnell und komplex reden wie immer - alle sollen sich gefälligst anpassen. „Wie bitte?“', frage ich. Und sie erklären es genauso noch einmal, nur schneller, weil es ja das zweite Mal ist, dass sie es sagen.

Frage: Ist die brutale Ganzheitsmethode der mathematischen Universitätslehre denn sinnvoll? Gehen Sie denn einfach so nach China, wo sie dann nur chinesisch sprechen? Wer macht das so - ohne Vorbereitung? Gehen Sie denn wirklich nur deshalb nach China, um die Sprache in vielen Jahren zu erlernen? Nein - Sie gehen dorthin, weil Sie die Kultur dort lieben und an ihr teilhaben wollen. Sie lieben das, was auf Chinesisch gesagt und diskutiert werden kann. Sie lieben die Erweiterung des Gesichtsfeldes, nicht das Erlernen der Sprache an sich.

Und dann kommen die Schüler, die die mathematischen Schönheiten von der Schule her vielleicht schon lieben gelernt haben, in die Universität und werden von Anfang an fast komplett von Schönheiten und wichtigen Erkenntnissen ausgeschlossen. Sie lernen das, was sie schon lieben, drakonisch noch einmal in einer harten unnahbaren Sprache. Niemand macht ihnen Hoffnung, dass ein Schönes oder Wichtiges dereinst auf sie warten würde. Es steht jedoch nur wie selbstverständlich, aber durchaus zweifelhaft im Raum.

Ich will damit etwas sagen: Das Grundübel der Mathematik ist das ostentativ nichtkümmernde Lehren einer dann ganz kalt erscheinenden Sprache, für die sich der Lernende nur schwer erwärmt. Das Lehren der Sprache steht absolut im Vordergrund, danach das Errichten nüchterner Gedankengebäude. Von Schönheit ist die Rede fast nie. Deren Existenz wird behauptet, aber sie wird nicht enthüllt. Man sieht sie wohl nach dem Doktor erst nur.

Die Veranstaltungen zum Mathematikjahr 2008 zeigen, dass sich Schönheit und Nutzen der Mathematik in Herzen senken lassen. Die Menschen öffnen sich bereitwillig, wenn die Mathematik so zu ihnen spricht, dass sie sie gebrochen verstehen. Sie beginnen die Mathematik zu lieben, so, wie wenn sich ein Franzose rührend um eine Amerikanerin in Paris kümmern würde. Sie lieben die Mathematik, wenn diese sich bemüht, in der Sprache der Menschen zu sprechen. Sie lieben sie vor allem deshalb, weil die Mathematik Wichtiges zu ihnen spricht.
Das tut die universitäre Lehre nicht. Um den Stoff rankt sich ein einziges ratloses „Warum?“” der Studenten. Braucht man das? Warum? Und die Antwort ist immer wieder: „Der Stoff ist eigentlich nur der Text, an dessen Beispiel die Sprache gelernt werden muss. Es geht um den Erwerb der Sprachkenntnis allein. Der Stoff ist sekundär. Später, wenn die Sprache gelernt ist, kann eventuell auch damit Wichtiges gesagt werden. Es geht um Verfahren, Beweistechniken, Denkschemata und Invariantenstudium. Erst die Sprache und nur die Sprache!" Der aber, der lernt, liebt die Aussagen in der Sprache, nicht die Sprache an sich. Die Mathematik leidet denn auch nicht an ihrer Sprachbarriere an sich, nur an der viel zu starken Überbetonung der Sprache gegenüber dem Wichtigen, das man sich mit ihr erschließt.

Damit ich nicht nur alles analysierend zersetze - ein Vorschlag. So würde ich es tun, wenn man mich ließe: Im ersten Semester Mathematik gibt es drei Grundvorlesungen.

\section{- Analysis I}

- Geschichte der Mathematik

- Jede Woche eine Übersicht über die wichtigen Grundaussagen/Ergebnisse/Studienobjekte/Anwendungen der Zweige der Mathematik: Zahlentheorie, Funktionentheorie, Algebra, Statistik, Numerik, Topologie, Differentialgleichungen ...

Ich belasse es einmal bei diesem ersten Vorschlag.

Ich ahne oder weiß schon, was Sie sagen ... zu viel „Blabla“ und die Studenten lernen wieder etwas Weiches wie bloße schöne Ergebnisse, anstatt „Beweisen, Beweisen, Beweisen“. Warum denn nicht Geschichte der Mathematik? Die prägt ja Sie und mich. Und wer sie gar nicht kennt, weiß nicht, wie ungut sie uns heute prägt. In der jüngeren Geschichte der Mathematik spielt „Nicolas Bourbaki“ nämlich in den meisten von Ihnen eine wichtige Rolle und treibt dort sein Unwesen. Seit 1934 hat Bourbaki den streng logischen Stil geprägt. Er führte die rigide Schreibweise ein, um die Worte und Erklärungen als Redundanz loszuwerden. Er predigte axiomatischen Stil und aggressiven Verzicht auf jedes Zugeständnis an irgendwelche Leser, Studenten und Professoren. Mathematik als strenges überkomplexes Mega-Gebäude - ohne Ansehen des Menschen. Pierre Cartier, der zur Bourbaki-Gruppe gehörte, soll gesagt haben, dieses Gebäude sei letztendlich wie ein Dinosaurier geworden, dessen Kopf zu weit vom Schwanze sei. Niemand überblickte das Gebäude am Ende noch ganz und man hörte auf zu bauen. In den Vorlesungen werden nun alle diese Hallen und Wände gezeigt, so weit sie fertig geworden sind. Das Wichtige aber sind nicht die ehrwürdigen, 
streng konstruierten Gebäude, sondern das triviale Leben darin. Das Abstrakte, was die Vertreter der reinen Lehre so abweisend predigen, ist eine „Errungenschaft“ der jüngsten Vergangenheit, sonst nichts! Soll oder muss das ewig so bleiben? Hat sich nicht auch die Psychologie inzwischen wieder dem Menschen genähert?

\section{Weltläufigkeit}

Das Leben mit der Mathematik sind insbesondere ihre Anwendungen. Die sind zunächst selten Teil der systematischen Grundlegung des Theoriegebäudes. Zuerst ist man an der Lösung von Problemen des Alltags interessiert, nicht an einer Theorie. Wie die Lösung erzielt wird, ist zweirangig. Der Anfangserfolg ist für den strengen Mathematiker meist wissenschaftlich trivial. Der angewandte Mathematiker nutzt eben irgendwo herumliegende Methoden, um schnell ein Ergebnis in der Praxis zu erzielen.

Ich war seit 1977 in der so beschimpften Angewandten Mathematik tätig und habe mir oft das Wort Dünnbrettbohrer anhören dürfen, was dann als Rote Laterne an die Informatiker ging. (Bei IBM sind die Puristen die Mitarbeiter, die sich mit Hardware befassen! Und dann gab es Software, so weiches, untechnisches, künstliches Zeug! Und heute Service und Beratung! „Methoden“!)

Das Angewandte aber ist das Wichtige im Leben der Nicht-Mathematiker und außerdem die entscheidende Schnittstelle, die Wissenschaft zu erneuern und am Leben zu erhalten. Besonders die Wissenschaftler sagen es Einstein nach: „Das Wichtigste ist, die guten Fragen zu stellen." Die guten Fragen aber stellt das Leben, eher selten die Wissenschaft. Das Leben spricht nicht die Sprache der Mathematik, sondern die eigene und - Verzeihung - die wichtigere und vor allem mächtigere. Die Fragen des Lebens müssen von der Mathematik aufgenommen werden.

Das geschieht nicht mit dem nötigen Ernst und vor allem nicht mit dem gebührenden Respekt dem Leben gegenüber. Es erinnert an das, was die Shareholder-Value-Manager gerade tun: Sie erzwingen die unnatürlich gepresste Abbildung des gesamten Wirtschaftslebens in bloße Zahlentabellen. Man zwingt das Leben Zahl zu sein, weil es dann leicht regiert werden kann. Die Mathematik aber verweigert sich ähnlich allem, was nicht mathematisches Modell ist. Beides ist falsch. Wissenschaft dient dem Leben. Nicht der Wissenschaft selbst und auch nicht dem bloßem Nutzen oder einzelnen Machthabern nein, dem Leben und den Menschen insgesamt.
Deshalb muss vielleicht nicht die Mathematik, aber der Mathematiker als solcher die Sprache des Lebens ebenso flüssig verstehen und eine Brücke sein. Das Leben braucht die Mathematik. So herum, und nicht: Der Mathematiker braucht Drittmittelgeld vom Leben. Das Leben schenkt uns die Fragen und die geliebten Probleme. Früher kamen die Fragen aus der Physik - daraus entstand ja zum guten Teil die heutige Mathematik. Aber sie befruchtete die Mathematik so sehr, dass es bald reine Mathematiker gab, die sich nur der Mathematik widmeten und nicht mehr den Lebensfragen da draußen. (In Großunternehmen gibt es auch Zahlenmenschen, die sonst nichts anderes sehen. In Kirchen gibt es Dogmatiker, die das Leben unterdrücken wollen. Immer geht es um das Primat eines Wissensgebietes gegenüber dem Leben an sich.).

Mathematik ist eine Sprache unter vielen. Wir Mathematiker halten sie für die Wichtigste. Wir sagen heute allgemein: „Mathematik ist die Schlüsseltechnologie." Ich habe heute, nach so langer Zeit, subjektiv das Gefühl, diesen Satz vielleicht sogar selbst erfunden, mindestens aber verbreitet zu haben. Rainer Janssen (mein damaliger Manager bei IBM und heute $\mathrm{ClO}$ der Münchner Rück) und ich schrieben im Jahre 1991 einen Artikel mit dem Titel: Mathematik: Esoterik oder Schlüsseltechnologie? Er erschien in DAS MAGAZIN (Wissenschaftszentrum NRW) im Jahre 1992. Dort stand ich noch echt unter meinem Zorn, als Angewandeter Mathematiker ein triviales Nichts zu sein, welches inexakte Methoden in der Industrie ganz ohne Beweis benutzt und mit Millioneneinsparungen protzt, obwohl gar nicht bewiesen werden kann, dass die gewählte Methode die allerbeste gewesen ist. Damals forderten Rainer Janßen und ich, dass Mathematik sich als Schlüsseltechnologie begreifen sollte. Wir meinten nicht, dass sie eine solche schon wäre. Ich jedenfalls meinte schon gar nicht, dass Mathematik das Wichtigste von allem ist, obwohl wir das immer ein bisschen propagierten, um Dampf zu machen.

Wissen Sie: Ohne Biologie würden wir nicht leben und ohne Chemie schnell sterben, wovor uns die Medizin rettet, die auch am wichtigsten ist. Die Physik ist die Königin der Wissenschaften, ohne Computer und damit Informatik wären wir nichts - und darüber steht die Philosophie und noch darüber die Theologie und noch viel darüber die Psychologie, die alles erklärt und ganz oben die Wirtschaftstheorie, weil ohne Management bekanntlich nichts geschieht, aber durch Management geschieht zuviel, deshalb thront oben Jura, die Ordnung und wieder darüber die Rüstungsindustrie etc. ... Sie alle haben ihre Sprachen und ihre Beziehung zum Leben. Manche sind zu manchen Zeiten Schlüs- 
selwissenschaften, manche bestehen aus immer leereren Hallen, so wie verlassene Kirchen.

Ja, Mathematik ist eine Schlüsseltechnologie, aber eine unter recht vielen, die alle zusammen multi-kulturell ein Ganzes erschaffen können. Die Mathematik muss sich mit freudigem Herzen diesem Ganzen widmen - dem Leben. Sie muss weltläufig und weltzugewandt sein. Sie muss sich nach außen verpflichtet zeigen, den Menschen und dem Leben etwas Wichtiges zu sein und zu BRINGEN. Sie muss sich in allen Sprachen verstehen und mitteilen können. Sie muss das Wichtige des Lebens und des Menschen höher sehen als das interne Wichtige.

Die Menschen sagen, die Mathematiker säßen im Elfenbeinturm - sie kämen nicht raus. Ist das zuviel verlangt? Vielleicht. Aber warum fangen wir nicht an, das Leben hereinzulassen?

\section{Studienabbrecher und Didaktik}

Das Leben sind zuerst die Studenten, die wir allein mit der schrecklichen Sprache lassen. Sie brechen zu großen Teilen das Studium ab. Mein Sohn zum Beispiel ist richtig gut und studiert gerade Mathematik - alles wirklich gut. Ohne mich aber wäre er am Anfang des Studiums glatt untergegangen, er war wochenlang verzweifelt. Kann man also ohne einen Mathe-Prof zu Hause studieren? Diese Erlebnisse in seinem ersten Semester bis zum Vordiplom sind mir sehr nahe gegangen. (Ich habe eine lange Kolumne darüber im Informatik-Spektrum im Jahre 2007 geschrieben.) Ich habe mit meinem Sohn noch einmal körperlich leidend miterlebt, wie das Leben, der Gast oder der neue Jünger gegrüßt und behandelt werden.

Jetzt ist mein Sohn unseresgleichen, es geht ihm gut. Alle sind nett zu ihm. Aber bis dahin? Bis dahin haben ihn eben nur Assistenten gesehen? Er kam neulich nach Hause und sagte: „Es sind kaum noch welche übrig von denen, die anfingen. Das ist sehr schlimm, aber wenn du es positiv siehst, kannst du jetzt endlich gut arbeiten. Alle sind interessiert und machen die Aufgaben. Keine öden Seminarvorträge am Rande von komplettem Unwissen. Und jetzt reden die Professoren mit uns." Und die Professoren sind jetzt bestimmt stolz auf den verbliebenen kümmerlichen Rest und schwärmen von ihrem fabelhaften Nachwuchs, den sie heranbildeten. (??) Muss das alles zwingend so ablaufen?

Deshalb schlage ich vor, dem Studienanfänger erst einmal die Mathematik im Ergebnis zu zeigen, wie im Museum oder auf dem CeBITStand, wie im Mathematicum in Gießen, wie auf dem Mathe-Schiff des Mathematikjahres. Sehnsucht zum Mitmachen sollte Triebfeder sein können, Sehnsucht, später das Leben mitzuprägen. Keine Initiationsriten mit Durchhalteappellen: „Hart, lang, entbehrungsreich, aushalten, Wille, Nacht zum Tag." Das sagen die indischen Asketen auch so und wundern sich, dass niemand Sehnsucht nach Nagelbrettern hat. ,Irgendwann siehst du das Licht! Und du stehst über den anderen, die nur trivial leben!“ Bitte - das Licht kann auch anders gezeigt werden, und dann bleiben die Studenten auch da.

Die Didaktik wird oft als Technik gesehen, den Einstieg zu erleichtern. Aber es ist nicht das Problem, das Sprachelernen einfacher zu machen, sondern das Verständnis über das vorherige Verstehen und Würdigen der Ergebnisse der Mathematik zu erziehen.

Man lernt doch nicht einfach immer nur Bilder zu malen, bis man es kann, oder? Ich schaue mir alles erst in Kunstbüchern an. Man lernt doch nicht Schach durch gnadenloses Üben, oder? Ich sehe mir erst Weltmeister an ...

Wir sollten den Geist und die Frucht der Mathematik höher stellen als die rigide Sprache, das Produkt höher als die Produktionstechnik. Dann kommen wir wieder in die Nähe des Lebens. Das Produkt können wir allen anderen mental verkaufen, die Produktionstechnik nicht. Ach, wenn wir zuerst einmal den Hass auf das Wort „,verkaufen“ erlöschen lassen könnten. Verkaufen heißt nicht: Anbiedern ans Leben. Verkaufen soll sein: Dem Leben eine Freude machen.

Adresse des Autors

Prof. Dr. Gunter Dueck IBM Chief Technologist dueck@de.ibm.com 